\title{
Advocacia do paciente pelos enfermeiros de terapia intensiva: uma revisão
}

\section{integrativa da literatura}

\author{
Patient advocacy by intensive care nurses: na integrative literature review \\ Abogacia del paciente por enfermeras de cuidados intensivos: una revisión integradora de la
}

literatura

Recebido:10/05/2021 | Revisado: 19/05/2021 | Aceito: 23/05/2021 | Publicado: 08/06/2021

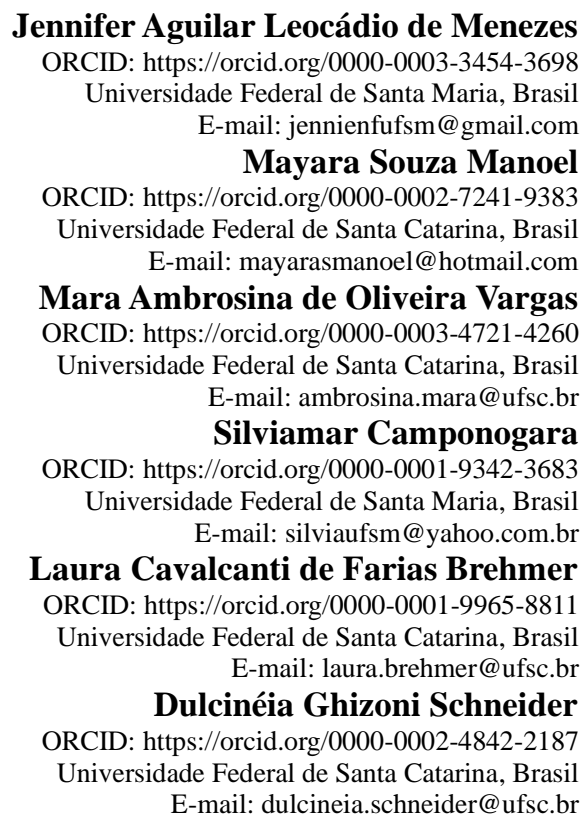

\begin{abstract}
Resumo
Objetivo: Identificar a produção científica acerca do tema advocacia do paciente por enfermeiros no contexto da terapia intensiva entre os anos de 2010 a 2020. Métodos: Revisão integrativa da literatura sobre o tema advocacia do paciente em unidade de terapia intensiva (UTI) entre os anos de 2010 a 2020, realizada nas bases de dados: Publisher Medline (PUBMED), Literatura Latino-Americana e do Caribe em Ciências da Saúde (LILACS), Cochrane Library e Web of Science, e também na biblioteca eletrônica Scientific Electronic Library Online (SCIELO) Com a busca nas base de dados, e na biblioteca eletrônica, foram encontradas 217 referências, das quais, 18 atenderam aos critérios de inclusão e exclusão da pesquisa. Resultado: Os 18 artigos selecionados foram categorizados conforme os temas identificados com maior recorrência, os quais consistem em: enfermeiro como defensor do paciente e família, barreiras para a defesa do paciente e enfermeiro como articulador entre equipe, paciente e família. Conclusão: Essa revisão possibilita levar ao meio científico, o que vem sendo estudado acerca da advocacia do paciente em UTI nos últimos 10 anos, em âmbito nacional e internacional, além de evidenciar à importância do enfermeiro enquanto defensor dos direitos do paciente e da família, explanar sobre as barreiras existentes para que a advocacia do paciente pelo enfermeiro intensivista ocorra de forma qualificada, e também demonstra como o enfermeiro executa o papel de intermediário entre seu paciente, família e equipe multiprofissional.
\end{abstract}

Palavras-chave: Advocacia em saúde; Defesa do paciente; Unidade de terapia intensiva; Enfermagem.

\begin{abstract}
Objective: To identify scientific production on the theme of patient advocacy by nurses in the context of intensive care between the years 2010 to 2020. Methods: Integrative literature review on the subject of patient advocacy in an intensive care unit (ICU) between the years 2010 to 2020, carried out in the databases: Publisher Medline (PUBMED), Latin American and Caribbean Literature in Health Sciences (LILACS), Cochrane Library and Web of Science, and also in the electronic library Scientific Electronic Library Online (SCIELO). electronic library, 217 references were found, of which, 18 met the inclusion and exclusion criteria of the research. Result: The 18 selected articles were categorized according to the themes identified with the greatest recurrence, which consist of: nurse as a defender of the patient and family, barriers for the defense of the patient and nurse as an articulator between team,
\end{abstract}


patient and family. Conclusion: This review makes it possible to take to the scientific community, what has been studied about the advocacy of patients in the ICU for the past 10 years, both nationally and internationally, in addition to highlighting the importance of nurses as defenders of the rights of the patient and the family, explain the existing barriers for the patient advocacy by the intensive care nurse to occur in a qualified way, and also demonstrates how the nurse performs the role of intermediary between his patient, family and multiprofessional team.

Keywords: Health advocacy; Defense of the patient; Intensive care unit; Nursing.

\section{Resumen}

Objetivo: Identificar la producción científica sobre el tema de la defensa del paciente por parte del enfermero en el contexto de cuidados intensivos entre los años 2010 a 2020.Métodos: Revisión bibliográfica integradora sobre el tema de la defensa del paciente en una unidad de cuidados intensivos (UCI) entre los años 2010 a 2020, realizada en las bases de datos: Editorial Medline (PUBMED), Literatura Latinoamericana y del Caribe en Ciencias de la Vida. Saúde (LILACS), Cochrane Library y Web of Science, y también en la biblioteca electrónica Scientific Electronic Library Online (SCIELO) Con la búsqueda en las bases de datos, y en la biblioteca electrónica se encontraron 217 referencias, de las cuales 18 cumplieron los criterios de inclusión y exclusión de la investigación. Resultado: Los 18 artículos seleccionados fueron categorizados según los temas identificados con mayor recurrencia, los cuales consisten en: enfermera como defensora del paciente y familia, barreras para la defensa del paciente y enfermera como articuladora entre equipo, paciente y familia. Conclusión: Esta revisión permite llevar al ámbito científico, lo que se ha estudiado sobre la defensa de los pacientes en la UCI durante los últimos 10 años, tanto a nivel nacional como internacional, además de resaltar la importancia del enfermero como defensor de los derechos. del paciente y la familia, explicar las barreras existentes para que la defensa del paciente por parte del enfermero de cuidados intensivos se produzca de manera calificada, y también demuestra cómo el enfermero desempeña el papel de intermediario entre su paciente, familia y equipo multiprofesional.

Palabras clave: Defensa de la salud; Defensa del paciente; Unidades de cuidados intensivos; Enfermería.

\section{Introdução}

O termo advocacia é proveniente do latim "advocatus", o qual consiste naquele que fornece evidências. Com isso, advocacia em enfermagem refere-se ao enfermeiro como fornecedor de subsídios com o objetivo de empoderar o paciente e seus familiares, para que esses possam tomar decisões conscientes acerca dos tratamentos de saúde (Vargas et al., 2019). Além disso, a advocacia também deve ser aplicada quando o paciente encontra-se impossibilitado de tomar suas próprias decisões, ficando sob responsabilidade do enfermeiro defender esse paciente perante a equipe de saúde (Luz et al., 2019, TomaschewskiBarlem et al., 2018).

A advocacia do paciente foi internacionalmente reconhecida como prática da enfermagem na década de 1970, através do Código de Ética da profissão determinado pelo Conselho Internacional de Enfermeiros (CIE) (Annas \& Healey, 1974), o que demonstra a atualidade do tema. No Brasil, a advocacia do paciente é apresentada como parte do exercício de cidadania exercido pelo enfermeiro, com o objetivo de respeitar os direitos humanos, inerente a ética profissional, e não comprometendo a atuação técnica da profissão (Tomaschewski-Barlem et al., 2018, Ventura, Mello, Andrade \& Mendes, 2012).

Em decorrência da contemporaneidade do tema advocacia do paciente na enfermagem, principalmente nacionalmente, encontram-se poucas publicações relativas a esse assunto. Uma das estratégias para tornar o assunto mais discutido na literatura nacional é através da realização de revisão integrativa da literatura internacional visando determinar o atual estado da arte da temática do conhecimento sobre a temática e realizar uma síntese e aprofundamento dos achados (TomaschewskiBarlem et al., 2018).

Particularmente, no que tange ao debate sobre advocacia do paciente no contexto da Unidade de Terapia Intensiva (UTI), essa discussão torna-se ainda mais pertinente. O estado grave dos pacientes, que necessitam de cuidados complexos, com uso de variedade de equipamentos e alta tecnologia, além do contato dos enfermeiros por longos períodos com os pacientes internados, tornam a UTI um ambiente de constante tomada de decisão, o que estimula o enfermeiro a exercer invariavelmente a advocacia do paciente (Vargas et al., 2019).

Devido a atualidade do tema advocacia do paciente em UTI, a deteç̧ão de escassez de publicações nacionais sobre o tema, evidencia-se a importância desse estudo como uma revisão integrativa da literatura, com o objetivo de melhor 
compreender o assunto, e principalmente, tornar mais evidente nacionalmente a advocacia do paciente em UTI. Questiona-se então: qual a produção científica acerca do tema advocacia do paciente por enfermeiros no contexto da terapia intensiva, entre os anos de 2010 a 2020? Com isso, o objetivo desse estudo foi: Identificar, através de uma revisão integrativa da literatura, a produção científica acerca do tema advocacia do paciente por enfermeiros no contexto da terapia intensiva, entre os anos de 2010 a 2020.

\section{Metodologia}

Trata-se de uma revisão integrativa da literatura, a qual permite a compreensão completa de um fenômeno analisado, pois determina o atual conhecimento sobre uma temática específica, possibilitando a síntese do conhecimento com o objetivo de realizar a prática baseada em evidências (Tomaschewski-Barlem et al., 2018).

Buscando seguir um rigor metodológico, foram seguidos os seis passos para a elaboração de uma revisão integrativa, descritos por Souza, Silva e Carvalho (2010), os quais consistem em: 1) Elaboração da pergunta norteadora; 2) Busca ou amostragem na literatura; 3) Coleta de dados; 4) Análise crítica; 5) Discussão dos resultados; 6) Apresentação da revisão integrativa (Souza, Silva \& Carvalho, 2010).

$\mathrm{Na}$ primeira etapa foi realizada a elaboração da pergunta norteadora de revisão, a partir da estratégia PICO, onde se considerou $\mathrm{P}$ (paciente) pacientes internados na unidade de terapia intensiva I (intervenção) Advocacia do paciente $\mathrm{C}$ (controle) não se aplica O (resultado) o exercício da advocacia do paciente pelos enfermeiros intensivistas. Sendo assim, obteve-se a seguinte pergunta: qual a produção científica acerca do tema advocacia do paciente por enfermeiros no contexto da terapia intensiva, entre os anos de 2010 a 2020 ?

Na segunda etapa, foi realizada a busca nas bases de dados, as quais consistiram em: Publisher Medline (PUBMED), Literatura Latino-Americana e do Caribe em Ciências da Saúde (LILACS), Cochrane Library e Web of Science, e também na biblioteca eletrônica Scientific Electronic Library Online (SCIELO). A busca em base de dados foi realizada com descritores de acordo com a classificação dos Descritores em Ciências da Saúde (DECS) da biblioteca virtual em saúde, os quais foram: Patient Advocacy (AND) Nursing (AND) Intensive Care Units. Além disso, nas bases de dados PUBMED e LILACS, e na biblioteca eletrônica SCIELO, além dos descritores foi possível utilizar sinônimos, resultando na seguinte sintaxe de busca: Intensive Care Units (OR) Intensive Care Unit (OR) ICU (OR) Critical Care (OR) Critical Care Unit (OR) Intensive Care (OR) Surgical Intensive Care (AND) Nursing (AND) Patient Advocacy (OR) Clinical Ombudsman (OR) Patient Ombudsman (OR) Patient Ombudsmen (OR) Patient Representative (OR) Patient Representatives.

Para inclusão dos estudos pesquisados estabeleceu-se como critério: a) Estudos publicados entre 2010 e 2020 e que abordem a advocacia do paciente pelo enfermeiro intensivista. Os critérios de exclusão de estudos foram: a) Artigos publicados em outros meios de comunicação que não periódicos científicos; b) Estudos na modalidade de cartas, resenhas e editoriais; c) Estudos duplicados; d) Estudos não disponíveis por completo.

Na terceira etapa, realizada em 17 de março de 2021, foi efetivada a busca nas bases de dados e biblioteca eletrônica. A partir da aplicação dos descritores, foram encontradas 217 referências, após a aplicação do filtro para os anos de 2010 a 2020 foram detectadas 92 referências. Justifica-se o recorte temporal como forma de obter uma quantidade considerável de estudos para análise, o que permite mapear como a temática vem sendo abordada nos últimos anos, e assim encontrar as lacunas no conhecimento para desenvolver futuros estudos.

$\mathrm{Na}$ quarta etapa, a partir da leitura do título e resumo dos estudos encontrados, iniciou-se a seleção dos mesmos. Com a aplicação dos critérios de exclusão, foram detectados 8 estudos duplicados, 66 estudos que não contemplavam o escopo da pesquisa, resultando em 18 estudos selecionados para serem lidos na íntegra. Após a leitura integral dos estudos, por contemplarem os objetivos propostos, todos foram selecionados para compor esta revisão integrativa da literatura. Além disso, 
na quarta etapa, a partir da leitura, foram obtidas algumas informações como: tipo e ano de publicação, título/autores, país/idioma, descritores/palavras-chave, base de dados/biblioteca de localização dos artigos, abordagem da pesquisa, objetivos, método, resultados, conclusões e observações. Estas foram organizadas e analisadas criticamente através do Microsoft Excel ${ }^{\circledR}$.

Sendo assim, a Figura 1 ilustra o fluxograma do itinerário metodológico até a conclusão da quarta etapa. No que diz respeito à quinta etapa, discussão dos resultados e a sexta etapa, apresentação da revisão integrativa, estas serão abordadas nos próximos itens desse artigo.

Figura 1 - Fluxograma do itinerário metodológico.

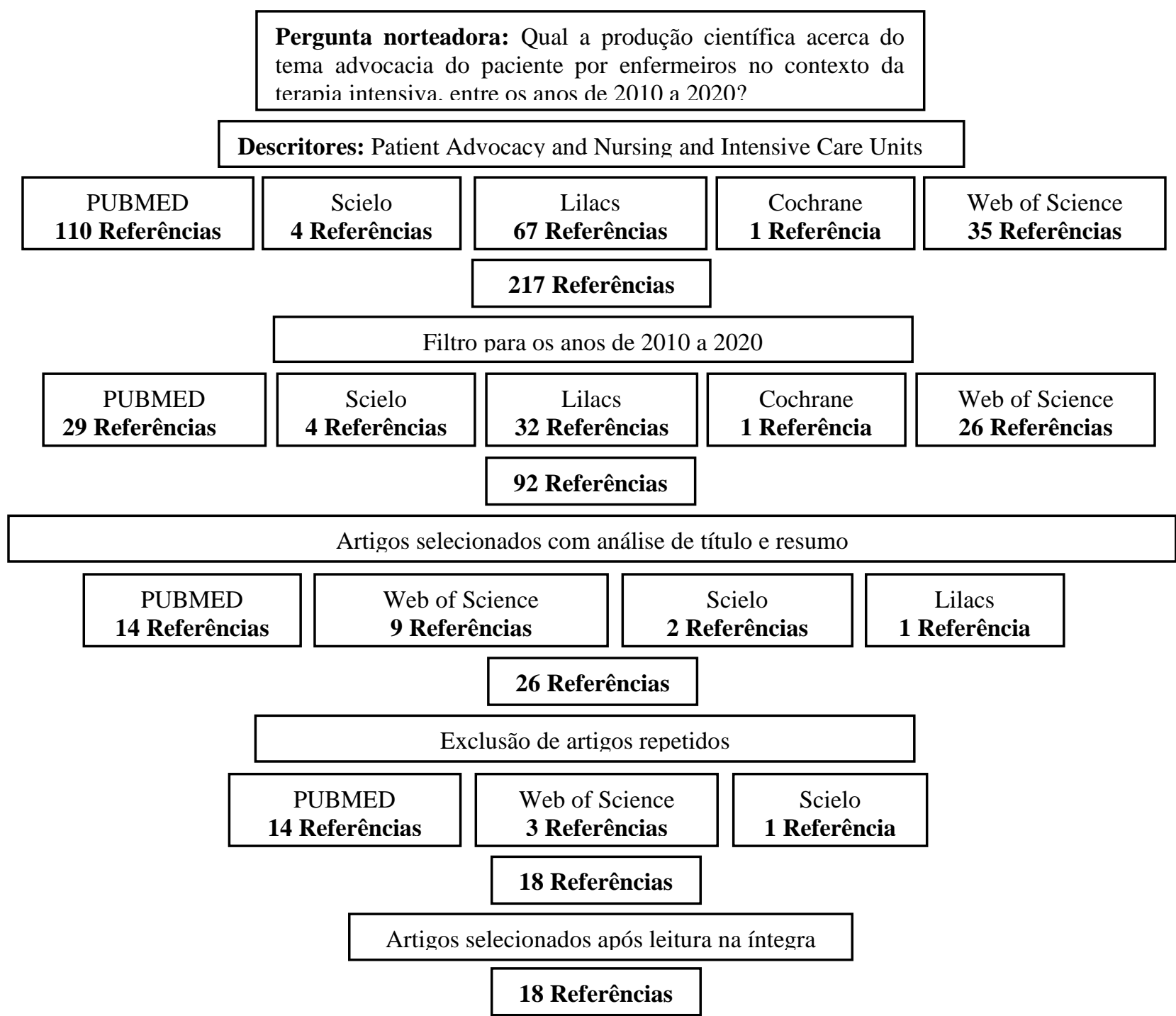

Fonte: Autores, embasado na busca em base de dados (17 de março de 2021).

Por se tratar de uma Revisão integrativa da literatura, o presente estudo não precisou ser submetido a um comitê de ética e pesquisa, devido ao fato de ter utilizado fontes de domínio público para sua realização. Foram seguidos rigorosamente os cuidados éticos na busca, análise, discussão e apresentação dos resultados.

\section{Resultados}

Dentre as publicações selecionadas, três são do ano de 2011, uma de 2012, duas de 2015, quatro de 2016, quatro de 2017, uma de 2018 e três do ano de 2019. Do total de referências selecionadas, quatorze foram obtidas na base de dados 
PUBMED, três da base de dados Web of Science e uma na biblioteca eletrônica Scielo. Todas as referências selecionadas encontravam-se na língua inglesa. Com relação aos países onde foram realizados os estudos, oito foram desenvolvidos nos Estados Unidos da América (EUA), três no Irã, um na Suécia, um no Reino Unido, um na África do Sul, um na Austrália, um na Arábia Saudita e dois no Brasil.

Sobre a metodologia dos estudos, doze utilizaram a abordagem qualitativa, três apresentaram metodologia qualitativa e quantitativa, dois utilizaram abordagem quantitativa, e um trata-se de uma reflexão teórica.

O Quadro 1 apresenta a caracterização das publicações.

Quadro 1 - Caracterização dos estudos sobre advocacia do paciente pelos enfermeiros de terapia intensiva, segundo: autor, ano de publicação, base de dados, país, título e abordagem metodológica.

\begin{tabular}{|c|c|c|c|c|}
\hline Autor/ano & Base de dados & País & Título & $\begin{array}{c}\text { Abordagem } \\
\text { metodológica }\end{array}$ \\
\hline Cypress, 2011 & PUBMED & EUA & $\begin{array}{l}\text { The lived ICU experience of nurses, patients and } \\
\text { family members: A phenomenological study } \\
\text { with Merleau-Pontian perspective }\end{array}$ & Abordagem Qualitativa \\
\hline Salmond, 2011 & PUBMED & EUA & $\begin{array}{l}\text { When the family member is a nurse: The role } \\
\text { and needs of nurse family members during } \\
\text { critical illness of a loved one }\end{array}$ & Abordagem Qualitativa \\
\hline $\begin{array}{l}\text { Engströma, Uusitaloa } \\
\text { \& Engström, } 2011\end{array}$ & PUBMED & Suécia & $\begin{array}{c}\text { Relatives' involvement in nursing care: A } \\
\text { qualitative study describing critical care nurses' } \\
\text { experiences }\end{array}$ & Abordagem Qualitativa \\
\hline Borowske, 2012 & PUBMED & EUA & $\begin{array}{l}\text { Straddling the Fence: ICU Nurses Advocating } \\
\text { for Hospice Care }\end{array}$ & Reflexão teórica \\
\hline $\begin{array}{c}\text { Ahluwalia, } \\
\text { Schreibeis-Baum, } \\
\text { Prendergast, Reinke, } \\
\text { \& Lorenz, } 2015\end{array}$ & PUBMED & EUA & $\begin{array}{l}\text { Nurses as intermediaries: how critical care } \\
\text { nurses perceive their role in family meetings }\end{array}$ & Abordagem Qualitativa \\
\hline Forsberg et al., 2015 & PUBMED & Reino Unido & $\begin{array}{c}\text { Attitudes towards organ donor advocacy among } \\
\text { Swedish intensive care nurses }\end{array}$ & $\begin{array}{c}\text { Abordagem Qualitativa } \\
\text { e Quantitativa }\end{array}$ \\
\hline Pasek \& Licata, 2016 & PUBMED & EUA & $\begin{array}{c}\text { Parent Advocacy Group for Events of } \\
\text { Resuscitation }\end{array}$ & $\begin{array}{c}\text { Abordagem Qualitativa } \\
\text { e Quantitativa }\end{array}$ \\
\hline $\begin{array}{l}\text { Beigzadeh, Borhani, } \\
\text { Ahmadi, \& } \\
\text { Abbaszadeh, } 2016\end{array}$ & Web of Science & Irã & $\begin{array}{c}\text { Nurses attribution to the role of patient advocacy } \\
\text { in intensive care units of hospitals of Tehran } \\
\text { University of Medical Sciences }\end{array}$ & $\begin{array}{c}\text { Abordagem Qualitativa } \\
\text { e Quantitativa }\end{array}$ \\
\hline $\begin{array}{l}\text { Davoodvand, } \\
\text { Abbaszadeh \& } \\
\text { Ahmadi, } 2016\end{array}$ & PUBMED & Irã & $\begin{array}{l}\text { Patient advocacy from the clinical nurses' } \\
\text { viewpoint: a qualitative study }\end{array}$ & Abordagem Qualitativa \\
\hline $\begin{array}{l}\text { Pecanac \& Schwarze, } \\
2016\end{array}$ & PUBMED & EUA & $\begin{array}{l}\text { Conflict in the intensive care unit: Nursing } \\
\text { advocacy and surgical agency }\end{array}$ & Abordagem Qualitativa \\
\hline $\begin{array}{l}\text { Rout \& Brysiewicz, } \\
\qquad 2017\end{array}$ & PUBMED & África do Sul & $\begin{array}{l}\text { Exploring the role of the ICU nurse in the } \\
\text { antimicrobial stewardship team at a private } \\
\text { hospital in KwaZulu-Natal, South Africa }\end{array}$ & Abordagem Qualitativa \\
\hline Bagherian, Sabzevari, & Web of Science & Irã & Effects of technology on nursing care and caring & Abordagem Quantitativa \\
\hline
\end{tabular}




\begin{tabular}{|c|c|c|c|c|}
\hline $\begin{array}{c}\text { Mirzaei, \& } \\
\text { Ravari, } 2017\end{array}$ & & & $\begin{array}{c}\text { attributes of a sample of Iranian critical care } \\
\text { nurses }\end{array}$ & \\
\hline $\begin{array}{l}\text { Becker, Wright \& } \\
\text { Schmit, } 2017\end{array}$ & Web of Science & EUA & $\begin{array}{l}\text { Perceptions of dying well and distressing death } \\
\text { by acute care nurses }\end{array}$ & Abordagem Qualitativa \\
\hline $\begin{array}{l}\text { Mortell, Abdullah \& } \\
\text { Ahmad, } 2017\end{array}$ & PUBMED & Arábia Saudita & $\begin{array}{c}\text { Barriers deterring patient Advocacy in a Saudi } \\
\text { Arabian critical care setting }\end{array}$ & Abordagem Qualitativa \\
\hline $\begin{array}{c}\text { Jakimowicz, Perry \& } \\
\text { Lewis, } 2018\end{array}$ & PUBMED & Austrália & $\begin{array}{c}\text { Insights on compassion and patient-centred } \\
\text { nursing in intensive care: A constructivist } \\
\text { grounded theory }\end{array}$ & Abordagem Qualitativa \\
\hline Vargas et al., 2019 & PUBMED & Brasil & Patient advocacy actions by intensivist nurses & Abordagem Quantitativa \\
\hline Luz et al., 2019 & Scielo & Brasil & $\begin{array}{c}\text { Advocacy in intensive care and hospitalization } \\
\text { by court order: what are the perspectives of } \\
\text { nurses? }\end{array}$ & Abordagem Qualitativa \\
\hline $\begin{array}{l}\text { Parsons \& Walters, } \\
\qquad 2019\end{array}$ & PUBMED & EUA & $\begin{array}{l}\text { Management Strategies in the Intensive Care } \\
\text { Unit to } \\
\text { Improve Psychosocial Outcomes }\end{array}$ & Abordagem Qualitativa \\
\hline
\end{tabular}

Fonte: Autores, embasado na busca em base de dados (17 de março de 2021).

Após a leitura e análise na integra das referências selecionadas, emergiram três categorias de análise, quais sejam: Enfermeiro como defensor do paciente e família; Barreiras para a defesa do paciente; Enfermeiro como articulador entre equipe, paciente e família. Essas categorias são apresentadas respectivamente conforme a perspectiva de cada autor nos quadros $2,3,4$.

Quadro 2 - Resultados dos artigos analisados na íntegra agrupados na categoria: Enfermeiro como defensor do paciente e família.

\begin{tabular}{|c|c|}
\hline \multicolumn{2}{|r|}{ ENFERMEIRO COMO DEFENSOR DO PACIENTE E FAMÍLIA } \\
\hline Cypress, 2011 & $\begin{array}{l}\text { - Há influência mútua entre a família, paciente e enfermeiros durante uma experiência crítica de doença, sendo necessária a } \\
\text { inclusão da família em todos os aspectos do cuidado. } \\
\text { - Enfermeiros perceberam a advocacia como um aspecto importante do seu papel na prestação de cuidados holísticos para os } \\
\text { doentes graves e seus familiares. } \\
\text { - Eles defendem o paciente e seus familiares: trabalhando com a família pelo bem-estar do paciente, advogando pela } \\
\text { privacidade e confidencialidade, advogando pelo paciente em questões éticas que incluem não ressuscitar, procuração de } \\
\text { assistência médica, testamentos em vida e diretivas avançadas, oferecendo informações ao paciente e familiar sobre a } \\
\text { condição atual, procedimentos, tratamento, equipamentos e linhas invasivas, prognóstico e números de telefone. }\end{array}$ \\
\hline Salmond, 2011 & $\begin{array}{l}\text { - Quando um membro da família é enfermeiro, esse identifica seu papel como primordial para proteger o paciente e os } \\
\text { demais familiares. } \\
\text { - A fim de fornecer cuidados centrados na família, o enfermeiro intensivista deve reconhecer as necessidades únicas do } \\
\text { membro da família/enfermeiro, permitindo que o membro da família/enfermeiro faça parte da equipe, facilitando o } \\
\text { acompanhamento contínuo pelo familiar/enfermeiro, buscando e esclarecendo informações para o familiar/enfermeiro. }\end{array}$ \\
\hline Engströma et al., 2011 & $\begin{array}{l}\text { - Os enfermeiros devem estabelecer uma relação de cuidado não apenas com o paciente mas também com a sua família, pois } \\
\text { estes poderiam contribuir com informações importantes e fornecer uma visão geral do paciente, a qual é essencial para } \\
\text { proporcionar o melhor atendimento ao paciente. } \\
\text { - O enfermeiro atua para que haja um equilíbrio entre o suporte dos parentes, recomendando que esses saiam um pouco do } \\
\text { hospital para descansar, alimentar-se e arejar, com o objetivo de manter o bem estar do paciente e dos parentes. } \\
\text { - Os enfermeiros zelam pela integridade do paciente, por exemplo ao pedir que parentes saíssem do quarto no momento do } \\
\text { banho, ficando atento se a presença de parentes está atrapalhando ou constrangendo o paciente. }\end{array}$ \\
\hline Borowske, 2012 & - Quando o médico não introduz o assunto de cuidados paliativos na UTI, é o enfermeiro quem aborda esse assunto junto à \\
\hline
\end{tabular}




\begin{tabular}{|c|c|}
\hline & com intuito de que paciente e família tenham processo de morte/morrer humanizado. \\
\hline Ahluwalia et al., 2015 & $\begin{array}{l}\text { - Um dos papeis do enfermeiro nas reuniões de família em UTI é ser o defensor, por ter contato constante com o paciente, } \\
\text { família e demais membros da equipe de saúde. }\end{array}$ \\
\hline Forsberg et al., 2015 & $\begin{array}{l}\text { - O cuidado de potenciais doadores de órgãos e seus familiares deve ser organizado de forma que promova a defesa de } \\
\text { direitos, como: salvaguardar e proteger ativa e pessoalmente a vontade e os desejos do potencial doador e dos parentes; } \\
\text { defender a doação de órgãos em nível hospitalar estrutural, em nível político e de pesquisa. } \\
\text { - É responsabilidade do enfermeiro representar o potencial doador de órgãos durante todo o processo, além de assegurar que } \\
\text { os colegas em seus turnos estejam cientes dos desejos do falecido ou do parente mais próximo. } \\
\text { - Enfermeiros com idade acima de } 50 \text { anos, e com experiência anterior em obter consentimento dos familiares em relação a } \\
\text { doação de órgãos e nos cuidados de pessoas com morte cerebral apresentaram maior defesa do doador. }\end{array}$ \\
\hline Pasek \& Licata, 2016 & $\begin{array}{l}\text { - Enfermeiros da UTI pediátrica são favoráveis a fornecer a opção de presença da família durante a ressuscitação } \\
\text { cardiopulmonar. }\end{array}$ \\
\hline $\begin{array}{l}\text { Beigzadeh et al., } \\
2016\end{array}$ & $\begin{array}{l}\text { - A atitude dos enfermeiros no papel de defesa do paciente foi positiva. } \\
\text { - } \quad \text { Enfermeiros com pós-graduação, casados, com mais idade e maior experiência profissional, sem fragilidade de saúde e que } \\
\text { apresentam situação econômica de média a alta, defendem mais o paciente. } \\
\text { - } \quad \text { Enfermeiros que receberam treinamento em cuidados de fim de vida para entender a advocacia realizam mais a defesa do } \\
\text { paciente. } \\
\text { - } \quad \text { o papel de advocacia dos enfermeiros leva a realização, promoção e satisfação no trabalho. } \\
\text { - } \quad \text { Exercer a defesa do paciente traz benefícios para pacientes, enfermeiros e todo o sistema de tratamento. }\end{array}$ \\
\hline $\begin{array}{l}\text { Davoodvand et al., } \\
\qquad 2016\end{array}$ & $\begin{array}{l}\text { - A defesa do paciente consistia em dois temas: empatia com o paciente (compreensão, simpatia e proximidade com o } \\
\text { paciente) e proteção dos pacientes (no atendimento, priorização da saúde, comprometimento com a conclusão do processo de } \\
\text { cuidado e proteção dos direitos dos pacientes). } \\
\text { - A necessidade de justiça está entre as necessidades humanas básicas, os enfermeiros estão em contato com os pacientes e } \\
\text { - } \quad \text { ous problemas, podendo oferecer justiça para os pacientes melhor do que qualquer outra pessoa. } \\
\text { - } \quad \text { A advocacia eficaz melhora a qualidade do atendimento ao paciente e enriquece a profissão de enfermagem. } \\
\text { - Os enfermeiros ao tentarem defender seus pacientes, sinalizam que um dia elas serão, também, pacientes. } \\
\text { - } \quad \text { Enfermeiros devem proteger os pacientes contra lesões e possíveis riscos físicos, mentais, deliberados, inadvertidos ou } \\
\text { devido a tratamento insuficiente ou a cuidados incorretos. Pois a doença reduz a força defensiva dos indivíduos, os pacientes } \\
\text { - } \quad \text { O enfermeiro cuida de indivíduos incapazes de defender seus direitos, ou não conhecem seus direitos e precisam de ajuda } \\
\text { - por meio de adequadas informações. } \\
\text { - O enfermeiro é responsável por proteger o paciente contra cuidados de saúde inadequados fornecidos por outros membros } \\
\text { da equipe de saúde. } \\
\text { - Os direitos dos pacientes, estado de saúde dos pacientes e a natureza da profissão de enfermagem foram os fatores mais } \\
\text { importantes declarados como motivo para proteger o paciente. } \\
\text { - Ao responder com empatia ao paciente e sua família, o enfermeiro pode ajudá-lo a adaptar-se ao seu problema. } \\
\text { A defesa do paciente fornecida pelos enfermeiros é baseada em seus valores pessoais e habilidades profissionais. }\end{array}$ \\
\hline $\begin{array}{l}\text { Pecanac \& Schwarze, } \\
2016\end{array}$ & $\begin{array}{l}\text { - Cabe ao enfermeiro a responsabilidade de apoiar a autonomia do paciente em relação às decisões de tratamento, atuar como } \\
\text { um intermediário entre o médico e o paciente e de apoiar o bem-estar do paciente. } \\
\text { - Enfermeiros capacitam os pacientes, garantindo que a voz do paciente seja ouvida durante a tomada de decisão do } \\
\text { tratamento. Enfermeiros devem fornecer informações adequadas para que o paciente faça a sua própria decisão e depois } \\
\text { apoiar a decisão do paciente. } \\
\text { - Enfermeiras podem defender o paciente ao ajudá-los a retomar sua independência, ao aliviar o sofrimento ou quando } \\
\text { promove o respeito ao paciente enquanto pessoa. Essas descrições reforçam a responsabilidade do enfermeiro como aquele } \\
\text { que trata o bem-estar do paciente e a essência de sua personalidade além do tratamento da doença. } \\
\text { - O enfermeiro exerce a defesa do paciente ao determinar com o paciente o significado pessoal para a experiência da doença, } \\
\text { sofrimento, ou morrer. Cada paciente tem um entendimento diferente de bem-estar, com base em sua construção pessoal, e }\end{array}$ \\
\hline
\end{tabular}


Research, Society and Development, v. 10, n. 6, e53110615968, 2021

(CC BY 4.0) | ISSN 2525-3409 | DOI: http://dx.doi.org/10.33448/rsd-v10i6.15968

\begin{tabular}{|c|c|}
\hline & $\begin{array}{l}\text { na experiência com a doença, bem como os efeitos da mesma no dia-a-dia. É só depois de estabelecer uma relação com o } \\
\text { paciente, que os enfermeiros podem compreender como defender suas necessidades e desejos. } \\
\text { - Os enfermeiros são líderes e defensores vigilantes na prestação de cuidados dignos e humanizados. }\end{array}$ \\
\hline $\begin{array}{l}\text { Rout \& Brysiewicz, } \\
\qquad 2017\end{array}$ & $\begin{array}{l}\text { O enfermeiro da UTI tem a responsabilidade de promover os interesses do paciente, e faz isso quando avalia a resposta do } \\
\text { paciente ao tratamento com antibióticos e discute com os médicos sobre a eficácia dos mesmos. Além disso, por acompanhar } \\
\text { o paciente diariamente, estão sempre atentos as mudanças clínicas. }\end{array}$ \\
\hline Bagherian et al., 2017 & $\begin{array}{l}\text { - Os enfermeiros do estudo tiveram uma atitude positiva em relação a defesa do paciente como integrante dos atributos de } \\
\text { cuidado. }\end{array}$ \\
\hline Becker et al., 2017 & $\begin{array}{l}\text { - Os enfermeiros elencaram três principais conceitos, sobre o que eles consideram necessário para que o processo de } \\
\text { morte/morrer seja humanizado, sendo eles: Apoio emocional e espiritual para pacientes e familiares; cuidado do paciente e } \\
\text { família e promoção de um ambiente de paz. } \\
\text { - Especificamente na UTI, estudo evidenciou que os enfermeiros prestam cuidados que atendem as necessidades e evitam o } \\
\text { sofrimento tanto do paciente quanto da família. } \\
\text { - Os enfermeiros da UTI demonstram compaixão, respeitando os desejos do paciente, facilitando a presença da família, } \\
\text { mantendo uma comunicação aberta sobre o estado de saúde do paciente, o que esperar e como confortar este. } \\
\text { Algumas ações dos enfermeiros, contribuem para que o processo de morte/morrer seja humanizado, sendo algumas delas: } \\
\text { comunicação com a equipe interdisciplinar e de enfermagem; prestação de um cuidado físico ideal; carinho e compaixão, } \\
\text { educação do paciente e família para enfrentar o processo de morte; apoio emocional; defesa do paciente e família; promoção } \\
\text { de ambiente pacífico. }\end{array}$ \\
\hline $\begin{array}{l}\text { Jakimowicz et al., } \\
\qquad 2018\end{array}$ & $\begin{array}{l}\text { - Alguns enfermeiros falaram do apoio que receberam da equipe médica e sua satisfação quando incluídos no planejamento } \\
\text { do tratamento do paciente. } \\
\text { - Os enfermeiros intensivistas precisam considerar todos os aspectos que envolvem o seu paciente, para assim conseguir } \\
\text { cumprir sua responsabilidade de defesa de direitos do mesmo, já que em diversas situações, os pacientes não conseguem } \\
\text { falar por si mesmos. }\end{array}$ \\
\hline $\begin{array}{l}\text { Parsons \& Walters, } \\
2019\end{array}$ & $\begin{array}{l}\text { - Os enfermeiros intensivistas contribuem diariamente com estratégias que ajudam os pacientes e familiares a se ajustarem, } \\
\text { para assim conseguir enfrentar as mais diversas doenças. } \\
\text { - Ao realizarem um cuidado integral que inclui: ajudar pacientes e familiares a lidar com o processo de doença, lesão ou } \\
\text { morte; realizar intervenções não farmacológicas junto aos familiares, com intuito de auxiliar no gerenciamento do estresse e } \\
\text { ansiedade; auxiliar no processo de reintegração a comunidade; praticar a advocacia, são intervenções que influenciam } \\
\text { positivamente nos aspectos psicossociais dos pacientes e familiares. }\end{array}$ \\
\hline Vargas et al., 2019 & $\begin{array}{l}\text { - Enfermeiros intensivistas consideram como advocacia do paciente, os cuidados que prestam com intuito de prevenir } \\
\text { agravos á saúde, quer sejam de origem física, mental, deliberação ou em decorrência da privação de tratamentos e cuidados } \\
\text { necessários. Ainda consideram que estas ações são de sua responsabilidade. } \\
\text { - A comunicação do enfermeiro, com objetivo de educar pacientes e familiares sobre seus deveres e direitos em relação a } \\
\text { internação, bem como as informações referentes a prognóstico, tratamento e potencial recuperação, também foi consideradas } \\
\text { como ações de advocacia. }\end{array}$ \\
\hline Luz et al., 2019 & $\begin{array}{l}\text { - Perante internação por ordem judicial em Unidade de terapia intensiva, o exercício da advocacia pelo enfermeiro } \\
\text { intensivista, encontra-se relacionada ao paciente que já está ocupando um leito e não tem condições de sair, e igualmente, } \\
\text { àquele que necessita de um leito e não consegue entrar. } \\
\text { - Enfermeiros defendem seus pacientes quando os informam sobre seus direitos, quando agem e falam sobre e em nome de } \\
\text { seus pacientes e familiares, esclarecendo sobre os tratamentos e a gravidade dos casos, prezando por um cuidado livre de } \\
\text { julgamentos e prejuízos para o paciente. }\end{array}$ \\
\hline
\end{tabular}

Fonte: Autores, embasado na busca em base de dados (17 de março de 2021).

Quadro 3 - Resultados dos artigos analisados na íntegra agrupados na categoria: Barreiras para a defesa do paciente. 


\section{BARREIRAS PARA A DEFESA DO PACIENTE}

\begin{tabular}{|c|c|}
\hline 2011 & $\begin{array}{l}\text { - O ambiente complexo da UTI e a falta de tempo são fatores que contribuem para que os enfermeiros não } \\
\text { envolvam os parentes nos cuidados. } \\
\text { - Proteger a integridade dos pacientes era uma das razões para limitar o envolvimento dos parentes nos cuidados. }\end{array}$ \\
\hline 2015 & $\begin{array}{l}\text { - Enfermeiros sinalizam sofrimento moral por não concordarem muitas vezes com o prognóstico, ou perceberem } \\
\text { discrepâncias e não conseguirem expressar isso nas reuniões familiares em UTI. } \\
\text { - Muitas vezes as decisões tomadas não estão de acordo com a vontade do paciente e os enfermeiros observam } \\
\text { uma barreira em expressar isso durante as reuniões de família da UTI. }\end{array}$ \\
\hline $\begin{array}{r}\text { Forsberg et } \\
2015\end{array}$ & $\begin{array}{l}\text { - Enfermeiros que trabalham em hospitais universitários, onde estão localizadas as unidades nacionais de } \\
\text { transplante, foram os menos positivos em relação à defesa da doação de órgãos. } \\
\text { - Cuidar de um paciente, possível doador de órgãos, é um trabalho exigente, e em grandes hospitais no contexto } \\
\text { em questão, existe uma da alta rotatividade de profissionais, e o emprego de enfermeiras jovens e inexperientes, } \\
\text { acabam por interferir no desejo de promover a doação de órgãos, e consequentemente, interfere na defesa dos } \\
\text { direitos do paciente, quando este expressa o seu desejo em ser doador. }\end{array}$ \\
\hline $\begin{array}{c}\text { Davoodvand et } \\
2016\end{array}$ & $\begin{array}{l}\text { - O objetivo da advocacia do paciente é fornecer cuidados de saúde de alta qualidade e proteger os direitos dos } \\
\text { clientes. No entanto, alguns fatores, como falta de competência e recursos, esgotamento, sofrimento profissional } \\
\text { e falta de dedicação à enfermagem, dificultam o alcance dessas metas. Por outro lado, esses fatores colocam os } \\
\text { clientes em risco, o que aumenta a importância da defesa do paciente. }\end{array}$ \\
\hline & 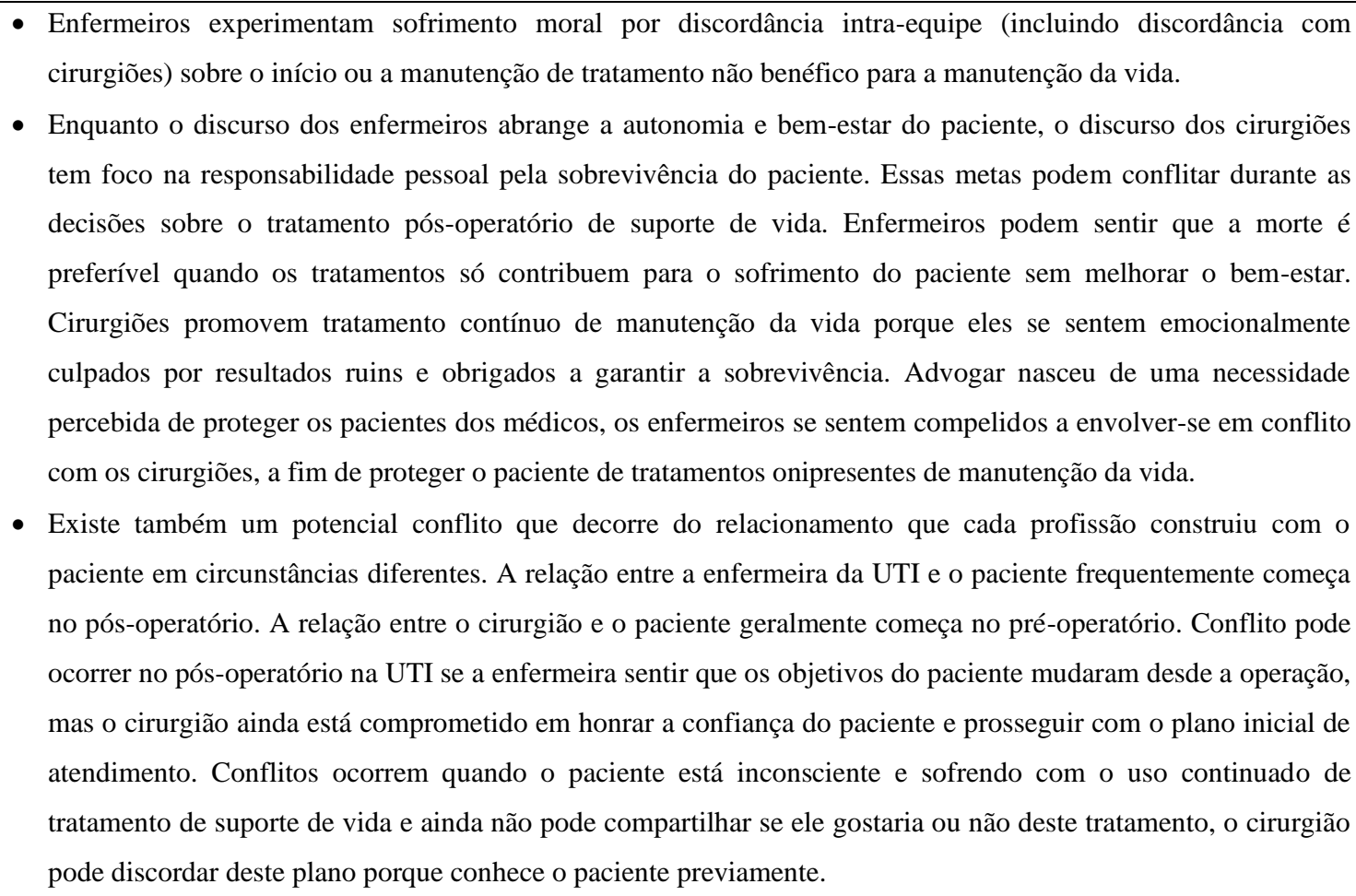 \\
\hline Mortell et al., 2017 & $\begin{array}{l}\text { - Questões relacionadas á gênero, cultura, educação, subjugação, clientelismo, falta de apoio institucional, } \\
\text { repercussões negativas e riscos associados á função, foram considerados fatores que afetam a capacidades dos } \\
\text { enfermeiros intensivistas da Arábia Saudita, de agir como defensores dos pacientes. } \\
\text { - A cultura organizacional é patriarcal e prevalece a autocracia médica. }\end{array}$ \\
\hline $\begin{array}{c}\text { Jakimowicz et al } \\
2018\end{array}$ & $\begin{array}{l}\text { - Alguns enfermeiros estavam insatisfeitos com a cultura médica, onde seu conhecimento não era reconhecido } \\
\text { nem usado, indicando aos participantes falta de respeito e compreensão. Como resultado, sentiram-se } \\
\text { desmotivadas e fatigadas. Os enfermeiros conheciam seus pacientes intimamente e poderiam atuar como sua } \\
\text { voz, mas às vezes isso não foi reconhecido por equipes médicas. }\end{array}$ \\
\hline
\end{tabular}




\begin{tabular}{|c|c|}
\hline & $\begin{array}{l}\text { - Defender o paciente foi recompensador e difícil. Atitudes familiares podem resultar em sentimentos de } \\
\text { incompreensão e desrespeito, relações familiares desafiadoras e defesa frustrada poderiam contribuir para que os } \\
\text { participantes sentissem fadiga e insatisfação, o que interrompeu a enfermagem centrada no paciente. } \\
\text { - Nessa situação, os enfermeiros acharam difícil fornecer a defesa do paciente, bem como manter sua própria } \\
\text { integridade pessoal e dignidade moral. } \\
\text { - Ser incapaz de cumprir seu papel de advocacia, impactou os sentimentos de satisfação profissional. }\end{array}$ \\
\hline Vargas et al., 2019 & $\begin{array}{l}\text { - Maior número de vínculos empregatícios, causam maior sofrimento, estresse e cansaço nos enfermeiros } \\
\text { intensivistas, ocasionando uma barreira ao exercício da advocacia do paciente por estes profissionais. } \\
\text { - A inexistência de reuniões no local de trabalho configura implicações negativas para a atuação do enfermeiro na } \\
\text { defesa do paciente. }\end{array}$ \\
\hline Luz et al., 2019 & $\begin{array}{l}\text { - As barreiras para exercer a advocacia do paciente, em situações de internação por ordem judicial, se apresentam } \\
\text { de três formas, sendo a primeira relacionada ao entendimento de que quem toma a decisão é o médico, por ser } \\
\text { responsável pelo gerenciamento dos leitos de UTI, segundo, pelo reconhecimento e acato da ordem judicial, e } \\
\text { terceiro, pela preocupação dos enfermeiros com uma possível punição e exposição. }\end{array}$ \\
\hline
\end{tabular}

Fonte: Autores, embasado na busca em base de dados (17 de março de 2021).

Quadro 4 - Resultados dos artigos analisados na íntegra agrupados na categoria: Enfermeiro como intermediário.

\begin{tabular}{|c|c|}
\hline \multicolumn{2}{|c|}{ ENFERMEIRO COMO ARTICULADOR ENTRE EQUIPE, PACIENTE E FAMÍLIA } \\
\hline Ahluwalia et al., & $\begin{array}{c}\text { Enfermeiros sentiam-se "presos no meio" ou "intermediários" na convivência com os pacientes, os familiares } \\
\text { dos pacientes e a equipe de atendimento, mas simultaneamente limitados na capacidade de participar } \\
\text { plenamente das reuniões familiares. }\end{array}$ \\
\hline Pecanac \& & $\begin{array}{c}\text { É crucial que os enfermeiros informem ao médico se o paciente não tem uma compreensão adequada do plano } \\
\text { de cuidados. Nesta função, o enfermeiro traduz informação do médico para o paciente ou vice-versa. Um } \\
\text { enfermeiro é um defensor no sentido de que ele age como intermediário entre o cliente e sua família, seu } \\
\text { médico e outros profissionais de saúde, com o objetivo final de beneficiar o cliente. }\end{array}$ \\
\hline Becker et al., 2017 2016 & $\begin{array}{c}\text { A enfermeira deve mediar com o médico para obter o que é necessário para o controle dos sintomas, e assim } \\
\text { contribuir para que o paciente em situação de final de vida, tenha uma morte pacífica. }\end{array}$ \\
\hline
\end{tabular}

Fonte: Autores, embasado na busca em base de dados (17 de março de 2021).

\section{Discussão}

A enfermagem exige de seus profissionais habilidades éticas, uma vez que os conhecimentos técnico e teórico, muitas vezes, não abordam os problemas dos pacientes e familiares. Portanto, o cuidado prestado pelo enfermeiro também é avaliado com base em sua comunicação, proteção, defesa e relacionamento com pacientes e familiares (Vargas et al., 2019, Ventura et al., 2012).

A temática da Advocacia do paciente no contexto da terapia intensiva, vem aos poucos ganhando espaço e sendo discutida em diversos países, tanto ocidentais (Ahluwalia et al., 2015, Becker et al., 2017, Forsberg et al., 2015, Pasek \& Licata, 2016, Pecanac \& Schwarze, 2016), quanto orientais (Bagherian et al., 2017, Beigzadeh et al., 2016, Davoodvand et al., 2016, Mortell et al., 2017), e é possível perceber diversas diferenças por conta dos contextos no qual estão inseridas, em alguns lugares sofrem forte influência de aspectos culturais e religiosos (Mortell et al., 2017).

No que diz respeito ao Brasil, compreende-se que a advocacia do paciente, foi proposta como um papel ético da prática profissional do enfermeiro, com o principal objetivo de zelar pelos direitos do paciente, contribuindo para a sua autonomia. Assim, essa defesa da enfermagem, pode ser definida como uma intervenção para ajudar os pacientes a obter 
serviços e benefícios que estão dentro de seus direitos, facilitando seu cuidado, tratamento e bem-estar como um todo (Luz et al., 2019, Vargas et al., 2019).

Enfermeiros no ambiente de cuidados intensivos desempenham um papel vital como advogados do paciente, pois buscam garantir que o tratamento, e cuidados prestados, sejam apropriados e seguros. No entanto, as barreiras que confrontam os enfermeiros como defensores, são frequentes e bem documentadas na literatura (Mortell et al., 2017, Vargas et al., 2019, Ventura et al., 2012).

A escassez de estudos brasileiros acerca da advocacia do paciente em UTI nos últimos dez anos, evidencia a importância de abordar o assunto nacionalmente. Além disso, as publicações internacionais sobre o tema também são escassas, uma vez que foram encontradas apenas 16 publicações sobre o tema, e a grande maioria se concentram na enfermagem norte americana.

A partir da análise dos artigos, identificaram-se três categorias recorrentes acerca da advocacia do paciente em UTI, sendo elas: $\mathrm{O}$ enfermeiro como defensor do paciente e família, barreiras para a defesa do paciente e o enfermeiro como articulador entre equipe, paciente e família.

Considerando o contexto da terapia intensiva, o enfermeiro julga importante o seu papel enquanto defensor do paciente e família, e considera como defesa do paciente algumas ações, como por exemplo: trabalhar em conjunto com a família para garantir o bem estar do paciente, advogar pela privacidade e confidencialidade, advogar pelo paciente em questões éticas, que incluem a não ressuscitação, testamentos em vida e diretivas avançadas, também considera, que defender o paciente e família, envolve a prestação de informações que dizem respeito sobre a condição atual de saúde, procedimentos a serem realizados e tratamento no geral (Cypress, 2011).

Sendo assim, o enfermeiro necessita estabelecer uma relação de cuidado não só com o paciente, mas também com a família, pois é esta que dará o suporte necessário para o doente, e também poderá contribuir com informações importantes que darão uma visão geral a respeito do paciente, contribuindo assim, para que o enfermeiro conheça melhor este indivíduo, e em uma possível necessidade de tomada de decisão, ou intervenção em defesa do paciente, este opte pelo melhor (Engström et al., 2011).

Os estudos ainda apontam outras situações em que se fazem necessárias que o enfermeiro intensivista atue como defensor dos pacientes e familiares, como por exemplo: nas reuniões de família (Ahluwalia et al., 2015), nos aspectos que envolvem o paciente possível doador de órgãos (Forsberg et al., 2015), nas questões que permeiam a presença ou não da família dentro da UTI (Pasek \& Licata, 2016), uso correto de antimicrobianos no tratamento de infecções (Rout \& Brysiewicz, 2017), pacientes em fim de vida (Becker et al., 2017, Borowske, 2012), como também, perante uma internação por ordem judicial na UTI (Luz et al., 2019).

Estudos sobre advocacia do paciente em outras unidades, citando aqui como exemplo, o centro de queimados, corrobora com os achados desta pesquisa, quando destaca que as principais ações de advocacia do paciente, estão relacionadas com a atitude de fornecer as mais diversas orientações, além de achar meios para protegê-lo, e também garantir a qualidade do cuidado, através da satisfação das necessidades do paciente, e da organização dos recursos da unidade (Nogario et al., 2015).

Ainda, estudos apontaram alguns benefícios do exercício da advocacia pelo enfermeiro, pois, ao realizar intervenções de defesa, estas influenciam positivamente nos aspectos psicossociais, tanto dos pacientes quanto de seus familiares (Parsons \& Walters, 2019). Além disso, existem benefícios para os próprios enfermeiros, gera a realização, promoção e satisfação no trabalho, ou seja, exercer a advocacia do paciente traz benefícios, para os pacientes e familiares, enfermeiros, e todo o sistema de tratamento (Beigzadeh et al., 2016). Em contrapartida, também pode causar sofrimento, conforme ficou evidenciado na categoria intitulada "Barreiras para a defesa do paciente". 
A advocacia do paciente tem entre seus objetivos, fornecer cuidados de saúde de alta qualidade, garantindo que os direitos e a autonomia do paciente sejam respeitados. Porém, alguns aspectos relevantes, como a falta de competência e recursos, esgotamento e sofrimento moral, bem como a falta de interesse dos próprios profissionais de enfermagem, pela profissão, constituem barreiras para que a defesa do paciente ocorra de forma efetiva (Davoodvand et al., 2016).

Os enfermeiros, também, identificaram como barreira, a interferência da família, pois apesar de os enfermeiros estimularem a participação dos familiares na tomada de decisões e nos cuidados (Engström et al., 2011), alguns familiares extrapolaram a linha tênue, entre auxiliar, e dificultar o trabalho da equipe de enfermagem (Jakimowicz et al., 2018, Pecanac \& Schwarze, 2016).

Além disso, outra barreira para a defesa do paciente e família, consiste na sobrecarga do enfermeiro ao ficar responsável por vários pacientes, somando além dos cuidados assistenciais diretos ao paciente, a realização de atividades de cunho gerencial que envolvem o ambiente da UTI (Davoodvand et al., 2016), pois por conta da alta complexidade, tanto da condição clínica do paciente, quanto de equipamentos, o enfermeiro precisa estar atento ao ambiente como um todo (Engström et al., 2011). Também detectou-se que enfermeiros de hospitais menores apresentam maior facilidade em exercer a defesa do paciente, do que os enfermeiros de grandes hospitais (Forsberg et al., 2015).

Estudo desenvolvido com enfermeiros intensivistas na Arábia Saudita, demonstrou o quanto questões relacionadas a gênero e cultura, podem constituir barreiras para o exercício da advocacia do paciente (Mortell et al., 2017).

$\mathrm{Na}$ Arábia saudita, a Enfermagem é considerada uma profissão nova, e ainda sofre grande resistência cultural (Mortell et al., 2017). Assim como em diversos outros países, na enfermagem, existe a predominância de indivíduos do sexo feminino, e na cultura oriental, o contato da mulher com homens que não sejam da sua família é inaceitável, e proibido. Ainda, as meninas são educadas desde cedo para não falarem fora de casa, ou seja, em público, um fator que as impede muitas vezes de falar em nome do paciente, quando este se encontra em riscos potenciais ou reais (Mortell et al., 2017).

Outro ponto relevante para discussão, diz respeito ao sofrimento moral vivenciado por enfermeiros ao tentar exercer a defesa do paciente (Ahluwalia et al., 2015). Esse ocorre devido a vários fatores, dentre eles, a discordância intraequipe, principalmente entre médicos e enfermeiros, a respeito da melhor conduta a ser tomada no tratamento do paciente (Pecanac \& Schwarze, 2016). Ainda dentro desse aspecto, considera-se que a cultura médica, constitui uma barreira e pode levar ao sofrimento moral, pois alguns enfermeiros relatam que seus conhecimentos científicos não são reconhecidos e nem utilizados, o que é considerado uma falta de respeito e compreensão, além de fazer com que os profissionais se sintam desmotivados e fatigados, e consequentemente não exercessem a advocacia do paciente de maneira efetiva (Jakimowicz et al., 2018).

Além disso, ficou evidente que um maior número de vínculos empregatícios, gera maior sofrimento, estresse e cansaço nos enfermeiros intensivistas, o que acaba ocasionando uma barreira (Vargas et al., 2019). Devido à baixa remuneração dos profissionais de Enfermagem, estes por diversas vezes, se obrigam a ter mais de um vínculo empregatício, para assim conseguir uma remuneração digna, o que pode colocar em risco a saúde do profissional, e também a do paciente, por conta do cansaço e estresse (Versa \& Matsuda, 2014).

Sendo assim, percebe-se que ainda são necessários muitos avanços relacionados ao exercício da advocacia do paciente por enfermeiros intensivistas, no contexto da terapia intensiva mundial, a fim de que as barreiras acima citadas, além de outras que ainda desconhecemos, possam ser superadas e os benefícios da advocacia, possam ser colhidos por todos os envolvidos no processo.

Conforme apontado em estudo, um dos principais papéis do enfermeiro intensivista, durante as reuniões de família, é atuar como defensor, pois este profissional mantém constante contato com o paciente, família e demais membros da equipe, podendo assim, ser capaz de atuar como um intermediário entre o paciente e demais sujeitos (Ahluwalia et al., 2015). 
Ainda, o enfermeiro é o primeiro defensor do paciente, sendo ele, o elo entre o paciente e o sistema de saúde como um todo, o que colabora para que ocorra uma advocacia eficaz, que consequentemente melhora a qualidade do atendimento, e enriquece a profissão de enfermagem (Davoodvand et al., 2016).

Também ficou evidente, que o enfermeiro atua como intermediário entre o paciente e o médico, quando percebe que o paciente não tem uma compreensão adequada a respeito do seu plano de cuidados, ou seja, ele faz um esclarecimento da informação do médico para o paciente, e vice-versa. Além disso, o enfermeiro também age como intermediário entre o paciente e seus familiares, buscando sempre, beneficiar o paciente (Pecanac \& Schwarze, 2016).

Ainda, o enfermeiro age como defensor do paciente, e ao mesmo tempo como articulador, no que diz respeito à terapêutica medicamentosa, quer seja para tratamento de infecções, quando o enfermeiro avalia a resposta do paciente ao tratamento com antibióticos, e discute a eficácia destes com o médico (Rout \& Brysiewicz, 2017), quanto em relação aos cuidados do paciente em final de vida, quando faz a mediação junto ao médico, para que o paciente receba medicação adequada para o controle de sintomas agudos, e assim, possa ter uma morte pacífica (Becker et al., 2017).

Sendo assim, percebe-se o importante papel do enfermeiro intensivista enquanto articulador, pois é ele quem está constantemente junto ao paciente, o que lhe permite conhecer os desejos e dúvidas deste, para assim conseguir intermediar tanto perante a família do indivíduo, quanto perante aos demais membros da equipe multiprofissional, que são responsáveis pelos cuidados desse paciente.

\section{Considerações Finais}

Os pacientes internados nas UTIs, na maioria das vezes, encontram-se impossibilitados de defender seus direitos, cabendo então ao enfermeiro, exercer a advocacia do paciente, no intuito de preservar a autonomia desse, garantindo que ele tenha todas as informações necessárias para que possa tomar as melhores decisões acerca do curso do tratamento clínico e plano de cuidados.

Essa revisão possibilita levar ao meio científico, o que vem sendo estudado acerca da advocacia do paciente em UTI nos últimos 10 anos, em âmbito nacional e internacional, além de evidenciar a importância do enfermeiro enquanto defensor dos direitos do paciente e da família, explanar sobre as barreiras existentes para que a advocacia do paciente pelo enfermeiro intensivista ocorra de forma qualificada, e também demonstra como o enfermeiro executa o papel de articulador entre seu paciente, família e equipe multiprofissional.

Considerando o número escasso de publicações encontradas, fica evidente a necessidade de estudos concernentes à advocacia do paciente em UTI, uma vez que, o exercício da defesa do paciente é capaz de trazer benefícios tanto para o paciente, que terá maior qualidade no cuidado, quanto para o profissional, que poderá ter maior realização e satisfação em seu trabalho. Porém, também são necessários mais estudos, que abordem as barreiras que implicam no exercício, ou não, da advocacia, para que a partir do conhecimento adquirido, possam ser elaboradas estratégias para romper com essas barreiras, e assim qualificar ainda mais o cuidado prestado pela enfermagem.

Além disso, sugere-se a realização de estudos que abordem a advocacia no contexto da terapia intensiva, considerando a perspectiva do paciente e seus familiares após vivenciarem uma internação neste ambiente. Neste sentido, também, conhecer a percepção de pacientes e familiares acerca do papel do enfermeiro como defensor dos seus direitos pode contribuir para o aprimoramento de estratégias e ações de advocacia.

\section{Referências}

Ahluwalia, S. C., Schreibeis-Baum, H., Prendergast, T. J., Reinke, L. F., \& Lorenz, K. A. (2015). Nurses as intermediaries: how critical care nurses perceive their role in family meetings. American Journal Of Critical Care, 25(1), 33-38, 2015. http://dx.doi.org/10.4037/ajcc2016653. 
Annas, G. J., \& Healey, J. (1974). The patient rights advocate. Journal of Nursing Administration, 4(3), 25-31.

Bagherian, B., Sabzevari, S., Mirzaei, T., \& Ravari, A. (2017). Effects of technology on nursing care and caring attributes of a sample of Iranian critical care nurses. Intensive And Critical Care Nursing, 39, 18-27. http://dx.doi.org/10.1016/j.iccn.2016.08.011.

Becker, C. A., Wright, G., \& Schmit, K. (2017). Perceptions of dying well and distressing death by acute care nurses. Applied Nursing Research, 33, 149-154. http://dx.doi.org/10.1016/j.apnr.2016.11.006.

Beigzadeh, M., Borhani, F., Ahmadi, F., \& Abbaszadeh, A. (2016). Nurses attribution to the role of patient advocacy in intensive care units of hospitals of Tehran University of Medical Sciences. Bioscience biotechnology research communications, 9(4), 884-890. http://bbrc.in/bbrc/2016Oct-DecPDF/BBRC 10_045.pdf.

Borowske, D. (2012). Straddling the Fence: ICU Nurses Advocating for Hospice Care. Critical Care Nursing Clinics Of North America, 24(1), 105-116. http://dx.doi.org/10.1016/j.ccell.2012.01.006.

Cypress, B. S. (2011). The lived ICU experience of nurses, patients and family members: A phenomenological study with Merleau-Pontian perspective. Intensive And Critical Care Nursing, 27(5), 273-280. http://dx.doi.org/10.1016/j.iccn.2011.08.001.

Davoodvand, S., Abbaszadeh, A., \& Ahmadi, F. (2016). Patient advocacy from the clinical nurses' viewpoint: a qualitative study. Journal Of Medical Ethics \& History Of Medicine, 9(5), 1-8. https://www.ncbi.nlm.nih.gov/pubmed/27471588.

Engström, B., Uusitalo, A., \& Engström, Å. (2011). Relatives' involvement in nursing care: A qualitative study describing critical care nurses’ experiences. Intensive And Critical Care Nursing, 27(1), 1-9. http://dx.doi.org/10.1016/j.iccn.2010.11.004.

Forsberg, A., Lennerling, A., Fridh, I., Rizell, M., Lovén, C., \& Flodén, A. (2015) Attitudes towards organ donor advocacy among Swedish intensive care nurses. Nursing In Critical Care, 20(3), 126-133. http://dx.doi.org/10.1111/nicc.12128.

Jakimowicz, S., Perry, L., \& Lewis, J. (2018). Insights on compassion and patient-centred nursing in intensive care: A constructivist grounded theory. Journal Of Clinical Nursing, 27(7-8), 1599-1611. http://dx.doi.org/10.1111/jocn.14231.

Luz, K. R. D., Vargas, M. A. D. O., Peter, E., Barlem, E., Viana, R. A. P. P., \& Ventura, C. A. A. (2019). Advocacy in intensive care and hospitalization by court order: what are the perspectives of nurses?. Texto \& Contexto-Enfermagem, 28.

Mortell, M., Abdullah, K. L., \& Ahmad, C. (2017). Barriers deterring patient advocacy in a Saudi Arabian critical care setting. British Journal of Nursing, 26(17), 965-971.

Nogario, A. C. D., Barlem, E. L. D., Tomaschewski-Barlem, J. G., Lunardi, V. L., Ramos, A. M., \& Oliveira, A. C. C. (2015). Nursing Actions in practicing inpatient advocacy in a Burn Unit. Revista da Escola de Enfermagem da Usp, 49(4), 0580-0588. http://dx.doi.org/10.1590/s0080-623420150000400007.

Parsons, L. C., \& Walters, M. A. (2019). Management Strategies in the Intensive Care Unit to Improve Psychosocial Outcomes. Critical care nursing clinics of North America, 31(4), 537-545.

Pasek, T. A., \& Licata, J. (2016). Parent Advocacy Group for Events of Resuscitation. Critical Care Nurse, 36(3), 58-64. http://dx.doi.org/10.4037/ $\operatorname{ccn} 2016759$.

Pecanac, K., \& Schwarze, M. L. (2016). Conflict in the intensive care unit: Nursing advocacy and surgical agency. Nursing Ethics, 25(1), 69-79. http://dx.doi.org/10.1177/0969733016638144.

Rout, J., \& Brysiewicz, P. (2017). Exploring the role of the ICU nurse in the antimicrobial stewardship team at a private hospital in KwaZulu-Natal, South Africa. South. Afr. j. crit. Care, 33(2), 46-50. http://dx.doi.org/10.7196/sajcc.2017.v33i2.331.

Salmond, S. W. (2011). When the family member is a nurse: The role and needs of nurse family members during critical illness of a loved one. Intensive And Critical Care Nursing, 27(1), 10-18. http://dx.doi.org/10.1016/j.iccn.2010.09.002.

Souza, M. T., Silva, M. D., \& Carvalho, R. (2010). Revisão integrativa: o que é e como fazer. Einstein, 8(1), 102-106. http://www.scielo.br/scielo.php?script=sci_arttext\&pid=S1679-45082010000100102\&lng=en\&nrm=iso.

Tomaschewski-Barlem, J. G., Lunardi, V. L., Barlem, E. L. D., Silveira, R. S. D., Ramos, A. M., \& Santos, J. M. D. (2018). Ações dos enfermeiros no exercício da advocacia do paciente: revisão integrativa. Texto contexto - enferm., 27(2), e0730014. http://www.scielo.br/scielo.php?script=sci_arttext\& pid=S0104-07072018000200501\&lng=pt\&nrm=isso.

Vargas, C. P., Vargas, M. A. D. O., Tomaschewski-Barlem, J. G., Ramos, F. R. S., Schneider, D. G., \& Camponogara, S. (2019). Patient advocacy actions by intensivist nurses. Revista da Escola de Enfermagem da USP, 53.

Ventura, C. A. A., Mello, D. F. D., Andrade, R. D., \& Mendes, I. A. C. (2012). Aliança da enfermagem com o usuário na defesa do SUS. Rev. bras. enferm., 65(6), 893-898. http://www.scielo.br/scielo.php?script=sci_arttext\&pid=S0034-71672012000600002\&lng=en\&nrm=iso.

Versa, G. L. G. S. \& Matsuda, L. M. (2014). Satisfação profissional da equipe de enfermagem intensivista de um hospital de ensino. Revista Enfermagem Uerj, 22(3), 409-415. 